\title{
La universidad y la efectividad del desarrollo comunitario: La promoción de la apropiación comunitaria en las Regiones Autónomas de Nicaragua ${ }^{1}$
}

\author{
The university and the effectiveness of community development: The promotion of community ownership \\ in the Nicaraguan Autonomous Regions
}

José Luis Saballos Velázquez²

\section{Resumen}

En más de 65 años de teorización y práctica, el desarrollo ha constituido uno de los conceptos más controvertidos y esquivos en su realización. Inicialmente, se consideraba que el desarrollo económico guiado por el libre mercado o el Estado, produciría una serie de beneficios que se distribuirían en cascada a todos los miembros de la sociedad, generando modernidad, el florecimiento de la democracia y un estado general de bienestar social. La imposibilidad de un rápido, significativo, equitativo y sostenido alivio de la pobreza ha replanteado en años recientes el poder que tiene la colectividad, los lazos afectivos y la identidad para un desarrollo inclusivo. Este trabajo resulta de una investigación doctoral en el campo de los estudios de desarrollo, que ha centrado su análisis en la apropiación comunitaria como una variable clave para determinar la efectividad de los procesos de acompañamiento al desarrollo comunitario. Basada en un estudio de casos múltiples (comunidades de Tuara, Españolina y El Carao), esta tesis doctoral resulta principalmente en una propuesta de modelo conceptual y metodológico de acompañamiento a la apropiación comunitaria, a partir de las perspectivas de la agencia colectiva, capital social y participación.

Palabras clave: Apropiación comunitaria; desarrollo comunitario; desarrollo con identidad; agencia colectiva; capital social y participación.

\section{Summary}

In little more than six decades of theory and practice, development has become one of the most controversial and illusive concepts in its achievement. At first, there was the firm belief that solely economic development would result in a trickle-down effect of benefits to the entire society, further leading to modernity, democracy and social wellbeing. The lack of a fast, significant, equitable and sustainable reduction of poverty has increasingly dragged attention to collectivity, affective ties and cultural identity for bringing about inclusive development. This doctoral research in the field of development studies focused on community ownership as a key variable for assessing the effectiveness of community development accompaniment processes. On the basis of a case study research methodology- carried in three communities (Tuara, Españolina and El Carao), a main outcome of this $\mathrm{PhD}$ thesis is a community ownership accompaniment model. This conceptual and methodological model is derived from the collective agency, social capital and participation theories.

\footnotetext{
1 XIV Congreso Latinoamericano y del Caribe de Extensión Universitaria y Segundo Congreso Centroamericano de Compromiso Social para la vinculación social de la universidad con la sociedad.

2 Doctor Internacional de Estudios de Desarrollo. Director de Cooperación Externa de la Universidad de las Regiones Autónomas de la Costa Caribe Nicaragüense.Correo: jose.saballos@uraccan.edu.ni ORCID: https://orcid.org/0000-0002-0160-1532
}

Recibido: 17/01/2018 - Aprobado: 05/04/2018 
Keywords: Community ownership; community development; development with identity; collective agency; social capital and participation.

\section{Introducción}

Esta investigación centra el análisis de la apropiación colectiva comunitaria como una variable clave de la efectividad del desarrollo comunitario. Este análisis referencia el proceso de acompañamiento al fortalecimiento de la autogestión comunitaria de los pueblos indígenas, afrodescendientes y mestizos llevado a cabo por la URACCAN en la Región Autónoma de la Costa Caribe Norte (RACCN). El modelo de Universidad Comunitaria Intercultural de la URACCAN surge para fortalecer la autonomía de los pueblos y comunidades indígenas, afrodescendientes y mestizas de las Regiones Autónomas de la Costa Caribe de Nicaragua.

Con este trabajo, por consiguiente, existe una preocupación sobre cómo contribuir a mejorar auto sosteniblemente las condiciones de vida a nivel comunitario de los pueblos indígenas, afrodescendientes y mestizos de las Regiones Autónomas de Nicaragua. Estos pueblos han sido afectados históricamente por racismo, discriminación, exclusión y debilitamiento de sus bases de cohesión e integración social.

En este sentido, se planteó un objetivo principal desde la perspectiva de la apropiación comunitaria: Determinar el alcance de la estrategia de acompañamiento a la autogestión comunitaria de la universidad en su contribución a la efectividad del desarrollo comunitario en el contexto de las Regiones Autónomas de Nicaragua. De este objetivo central, se derivan 5 objetivos secundarios, complementarios. Estos son:

1. Determinar las contribuciones de las acciones de acompañamiento a la autogestión comunitaria de la universidad a la participación, agencia colectiva y capital social en las comunidades de las regiones autónomas de Nicaragua.

2. Determinar la variación en la participación, agencia colectiva y capital social en las comunidades en relación a su etnicidad y los tiempos de acompañamiento de la Universidad.

3. Identificar los principales factores del contexto que afectan la participación, agencia colectiva y capital social en las comunidades acompañadas por la Universidad.

4. Del proceso de acompañamiento a la autogestión comunitaria de la Universidad, determinar las etapas del proceso de fortalecimiento de la apropiación comunitaria.

5. Identificar indicadores para la medición de los impactos de las acciones de desarrollo comunitario en la apropiación comunitaria en el contexto de las regiones autónomas de Nicaragua.

Esta investigación busca contrastar la siguiente hipótesis: Considerando la apropiación colectiva como la variable clave del desarrollo comunitario, la Universidad de las Regiones Autónomas de la Costa Caribe Nicaragüense (URACCAN), en su carácter de universidad comunitaria intercultural, dispone de capacidades específicas y ventajas comparativas para promover integralmente procesos de apropiación comunitaria que contribuya a una mayor efectividad del desarrollo comunitario.

\section{Revisión de la literatura}

Desde el modelo de universidad comunitaria intercultural de la URACCAN, se ha buscado como generar una propuesta de modelo de acompañamiento a la apropiación comunitaria que facilite el tránsito hacia una efectividad del desarrollo comunitario con identidad. La apropiación es un concepto que abre posibilidades de hacer del desarrollo un ejercicio efectivo de derechos humanos, ciudadanos y realización de autonomía de las personas especialmente en colectividad y desde sus culturas indígenas, afrodescendientes y mestizas. 
En Nicaragua, la Educación Superior tiene un desafío en constituirse en un sector importante y beligerante en la contribución a la generación de bienestar en toda la población, especialmente los sectores más pobres, marginados y excluidos. En este sentido, Unceta (2007) afirma: "la enseñanza superior constituye una herramienta fundamental para que las distintas sociedades puedan dotarse de los conocimientos y las capacidades que les permitan impulsar procesos de transformación y afrontar en mejores condiciones los retos del desarrollo en los más diversos campos" ( $\mathrm{p}, 43$ ).

Es por ello, que el análisis de la apropiación colectiva comunitaria se hace desde las perspectivas de la agencia colectiva, participación y capital social. Conforme estos ámbitos propuestos de la apropiación colectiva comunitaria, se han definido 5 variables: de la agencia colectiva, la variable de percepción colectiva de eficacia; de la participación, las variables de tipo de participación y nivel de liderazgo comunitario; y del capital social, las variables de confianza y nivel de acción colectiva. Véase Cuadro 1.

\section{Cuadro 1: Definiciones operativas de los ámbitos y variables}

\begin{tabular}{|c|c|c|}
\hline & & Definición Operativa \\
\hline \multirow{3}{*}{ 응 } & Agencia colectiva & $\begin{array}{l}\text { Es la habilidad que tienen las personas o grupos para plantearse objetivos que consideran importantes (cual- } \\
\text { quiera que sea), decidir sobre ellos y realizar las acciones necesarias para lograrlos (Sen, 1999). Se puede } \\
\text { ejercer en relación a ciertos objetivos, en relación a cierto espacio de interacción o bien en relación a cierta } \\
\text { fuerza coercitiva (Alkire, 2008). Esta involucra una capacidad para juzgar, actuar y provocar cambios (Urquijo } \\
\text { Angarita, 2007). }\end{array}$ \\
\hline & Capital social & $\begin{array}{l}\text { La valía de las relaciones sociales que posibilita la cooperación de beneficio mutuo dentro de los colectivos } \\
\text { (De Silva, Harpham, Huttly, Bartolini, \& Penny, 2007). Constituye una capacidad y activo de cooperación, re- } \\
\text { ciprocidad y confianza, que reside en las relaciones sociales y facilita el acceso a recursos mediante las redes } \\
\text { interpersonales y la acción colectiva organizada y sostenida. }\end{array}$ \\
\hline & Participación Comunitaria & $\begin{array}{l}\text { Un proceso y resultado social en que grupos con necesidades y un área geográfica de residencia en común } \\
\text { activamente ejercen acción colectiva en la identificación de intereses y prioridades, toma de decisión y el es- } \\
\text { tablecimiento de arreglos organizacionales de gestión (Butterfoss, 2006). Promueve valores, actitudes, cono- } \\
\text { cimientos y habilidades entre las personas, construyendo su capacidad individual y colectiva como agentes } \\
\text { de cambio (Bowen, 2008). Al mismo tiempo, moviliza energías, visiones, informaciones, experiencias, cono- } \\
\text { cimientos y recursos necesarios para lograr soluciones viables a problemas locales, una mejor distribución de } \\
\text { recursos y mejora de las condiciones de vida (Bowen, 2008). Contribuye a construir consensos, compromisos y } \\
\text { un sentido de apropiación comunitaria. }\end{array}$ \\
\hline \multirow{5}{*}{ 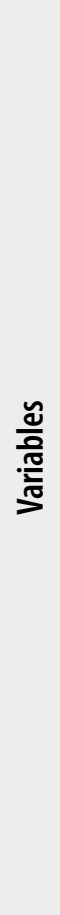 } & $\begin{array}{c}\text { Percepción colectiva de } \\
\text { eficacia }\end{array}$ & $\begin{array}{l}\text { Es la confianza que tiene un grupo o comunidad sobre sus capacidades para lograr los objetivos propuestos y } \\
\text { su nivel de control sobre los eventos que pueden surgir en dicho camino (Bandura, 1988). Esta posibilita una } \\
\text { acción colectiva capaz de transformar las relaciones y circunstancias que perpetúan la privación e inequidad } \\
\text { relacionadas a la pobreza y la exclusión social. }\end{array}$ \\
\hline & Tipo de participación & $\begin{array}{l}\text { En la participación comunitaria se encuentra el poder de emancipación de la gente común y corriente, de que } \\
\text { estos logren el control de su contexto, auto determinación y auto desarrollo (Geoghegan \& Powell, 2009). } \\
\text { Esta participación puede verse como la cúspide de un continuum de acumulación de capacidades, ejercicio de } \\
\text { poder de decisión y auto gestión de resultados deseados canalizados a través de la acción colectiva sostenida. } \\
\text { La participación en modo de empoderamiento, en vez de la participación informativa o consultativa, permite } \\
\text { responder mejor a las necesidades y demandas manifestadas por las comunidades acompañadas (Dahl- 0s- } \\
\text { tergaard et al., 2003). }\end{array}$ \\
\hline & $\begin{array}{l}\text { Nivel de liderazgo comuni- } \\
\text { tario }\end{array}$ & $\begin{array}{l}\text { Los líderes y lideresas tiene a su cargo el auto gobierno de la comunidad, cuyo desempeño democrático y } \\
\text { equitativo depende de su autoridad moral y una serie de capacidades- incluidas la capacidad de negociación, } \\
\text { movilización y gestión de recursos, mediación y resolución de conflictos, planificación y organización de la } \\
\text { participación en acción colectiva. El liderazgo local efectivo, combinado un adecuado sentido de comunidad, } \\
\text { es vital en la sostenibilidad de las estructuras organizativas orientadas a promover la participación comunitaria } \\
\text { (Nkansa \& Chapman, 2006). }\end{array}$ \\
\hline & Confianza & $\begin{array}{l}\text { Concepto abstracto que denota una actitud que permite la cesión voluntaria del control de recursos. Se refiere } \\
\text { igualmente a un aprendizaje social que se desarrolla a partir de experiencias positivas de reciprocidad y está } \\
\text { basada en expectativas mutuas de comportamiento. El grado de confianza existente entre individuos o grupos } \\
\text { tiene un fundamento histórico que se hace visible en la memoria social y colectiva (Nkansa \& Chapman, 2006). }\end{array}$ \\
\hline & Nivel de acción colectiva & $\begin{array}{l}\text { Actividades emprendidas por las personas, grupos y comunidades conforme intereses comunes y objetivos } \\
\text { compartidos. Se participa voluntaria u obligadamente. A mayor acción colectiva mayor nivel de capital social. }\end{array}$ \\
\hline
\end{tabular}


Estos ámbitos y variables corresponden al fenómeno social de la comunidad, especialmente en cuanto a su cultivo o fortalecimiento como espacio de realización de autonomía, planes de vida, anhelos y aspiraciones en colectividad e integración de esfuerzos, espíritu y visiones. Ante un desarrollo egoísta, etnocéntrico y desigual, la comunidad encierra el potencial de un desarrollo con identidad, inclusivo, solidario y sostenible. La comunidad es el camino al buen vivir. En cuanto a su definición, la comunidad es la arena en la que la gente adquiere sus más fundamentales y sustanciales experiencias de vida social fuera de los confinamientos del hogar, donde uno aprende y continua la práctica del ser social, donde uno adquiere la cultura, es decir, los símbolos requeridos para ser sociales y la capacidad de interpretar sus significados en la interacción con los otros (Cohen, 1985).

Así, la comunidad es un espacio de intersubjetividad cultural, es decir, de patrones de interpretación transmitidos generacionalmente y organizados lingüísticamente, desde donde se desarrolla la vinculación social y afectiva. La intersubjetividad se refiere a una conciencia de los otros como sujetos y una preocupación por su bienestar a partir de compartir un entendimiento del contexto histórico, lingüístico, social y político, y en base a ello tener experiencias similares (Cronick, 2002; O’Donnell, 2006). La intersubjetividad es un elemento fundamental del sentido de comunidad. El sentido de comunidad hace que las personas reconozcan la interdependencia que tienen con otras personas, que la expresen mediante acciones de empatía y reciprocidad y un sentimiento de pertenencia, especialmente en momentos que necesitan de algún apoyo (Lev-Wiesel, 2003).

\section{Materiales y métodos}

Estos ámbitos y variables de la apropiación comunitaria, se han escudriñado utilizando un estudio de casos múltiples, particularmente porque permite, entre otras cosas, refinar políticas, estrategias y expectativas institucionales. El estudio de casos múltiples han sido definidos como estudios que al utilizar los procesos de investigación cuantitativa, cualitativa o mixta, analizan profundamente una unidad para responder al planteamiento del problema, probar hipótesis y desarrollar alguna teoría (Sampieri, Fernández-Collado, \& Lucio, 2006, p. 224). Según Judith Bell, los estudios de caso permiten obtener una imagen tridimensional e ilustran las relaciones, los temas micro políticos y los modelos de influencia en un contexto determinado (Bell, 2002). Esta estrategia de investigación ha demostrado ser idónea para comprobar hipótesis, sustentadas en preguntas de investigación del tipo cómo y por qué, en un contexto donde el investigador tienen poco control sobre los acontecimientos, cuando el tema es contemporáneo y el fenómeno social no se distingue fácilmente de su contexto (Flyvbjerg, 2007; Sampieri et al., 2006; Yacuzzi, 2005; Yin, 2003).

Los estudios de caso seleccionados corresponden a la comunidad indígena miskita Tuara, la comunidad indígena mayangna la Españolina y la comunidad mestiza El Carao, que se ubican en los municipios de Puerto Cabezas, Bonanza y Siuna respectivamente en la RACCN. Estas comunidades fueron seleccionadas conforme 4 criterios principales: (i) que las comunidades fueran pobres y pertenecientes a un entorno rural; (ii) que las comunidades representasen de alguna forma la diversidad étnica de la población de las Regiones Autónomas; (iii) que la universidad hubiese implementado toda o casi todas las actividades de acompañamiento comunitario en las áreas de docencia, investigación y extensión; y (iv) que las comunidades representasen diferentes trayectorias de acompañamiento de la universidad no menor a 5 años.

En estas comunidades, se establecieron dos unidades de análisis: los líderes comunitarios (hombres y mujeres) e integrantes de organizaciones de base comunitarias. En la Universidad, la unidad de análisis fue el personal asignado al acompañamiento de estas comunidades en el periodo considerado de 2008 a 2012.

La revisión documental, la encuesta y entrevista son las principales técnicas aplicadas en estos estudios de caso. Tanto la encuesta como la entrevista se basaron principalmente en Cuestionario Integrado para la Medición del Capital Social del Banco Mundial. Este es un instrumento de aplicación internacional que ha sido validado en el contexto nicaragüense. 
Se debe mencionar que la investigación se fundamentó en el principio de consentimiento libre, previo e informado, en base al cual los líderes comunitarios aprobaron la realización de la etapa de campo de la investigación en sus comunidades. Esto conforme normativa de la URACCAN.

\section{Resultados}

La apropiación colectiva comunitaria se define como un estado de realización en el cual la comunidad, reflejando su voluntad en el liderazgo y organizaciones de base, ejerce su autonomía efectiva en su contexto de desarrollo (con identidad), incluyendo los distintos procesos e iniciativas de cooperación externa que afectan sus vidas.

En la práctica, la apropiación comunitaria implica un ejercicio efectivo de autoridad y liderazgo en los procesos de desarrollo con identidad de parte de la comunidad, al corresponder con:

1. Un liderazgo comunitario proactivo, transparente y democrático.

2. Una elevada percepción colectiva de eficacia sustentada en una apropiación de derechos y un florecimiento de capacidades de autogestión y diálogo desde lo endógeno.

3. Un amplio tejido de confianza. Una participación comunitaria activa, inclusiva y reflexiva.

4. Una acción colectiva solidaria conforme los propios planes de vida o desarrollo de la comunidad.

Consecuentemente, el acompañamiento a la apropiación comunitaria es una plataforma de diálogo horizontal y de acción conjunta, donde el actor externo tiene unos papeles trascendentales a jugar, elementos y principios fundamentales de trabajo a cumplir en su relacionamiento con las organizaciones de base y estructura de liderazgo de la comunidad, facilitando un cultivo o fortalecimiento de la agencia colectiva, participación, capital social y consecuentemente la apropiación comunitaria. La calidad de este tipo de acompañamiento está determinada por una capacidad organizacional de desempeñar efectivamente papeles como catalizadores, facilitadores, aliados estratégicos y partidarios de los actores comunitarios (Cuadro 2). Estos papeles son alternativos a los papeles tradicionales de salvadores, liberadores, modernizadores y proveedores de caridad, que se juegan desde la hegemonía del poder político, social, económico y/o tecnocrático, y resultan en paternalismo, subyugación, discriminación y denigración (Toomey, 2009).

Cuadro 2: Papeles a jugar en un acompañamiento a la apropiación comunitaria

\begin{tabular}{|l|l|l|l|}
\hline \multicolumn{1}{|c|}{ Catalizador } & \multicolumn{1}{|c|}{ Facilitador } & Aliado Estratégico & \multicolumn{1}{c|}{ Partidario } \\
\hline $\begin{array}{l}\text { Promover nuevas ideas, conocimientos, } \\
\text { percepciones y acciones que fortalezcan la } \\
\text { autonomía y auto determinación de la co- } \\
\text { munidad, mediante el fortalecimiento de } \\
\text { capacidades de identificación y solución } \\
\text { de problemas. }\end{array}$ & $\begin{array}{l}\text { Apoyando espacios de encuentro comunita- } \\
\text { rios de cara a la superación de diferencias, } \\
\text { participación de las más vulnerables movi- } \\
\text { lizaciones colectivas. }\end{array}$ & $\begin{array}{l}\text { Colaboración solidaria con la } \\
\text { comunidad conforme las priori- } \\
\text { dades, intereses y agenda de esta } \\
\text { en un marco de amistad. }\end{array}$ & $\begin{array}{l}\text { Apoyo político en procesos } \\
\text { de incidencia, visibilización } \\
\text { en la promoción del ejer- } \\
\text { cicio efectivo de derechos } \\
\text { ambientales, sociales, cultu- } \\
\text { rales, económicos y políticos } \\
\text { de las comunidades. }\end{array}$ \\
$\begin{array}{l}\text { Facilitar procesos de aprendizajes horizon- } \\
\text { tales, incluyendo intercambios con otros capacidades de facilita- } \\
\text { actores relevantes. }\end{array}$ & $\begin{array}{l}\text { nión propias organizaciones comu- } \\
\text { nitarias en áreas como toma de decisión, } \\
\text { gestión y administración de recursos, co- } \\
\text { municación y coordinación. }\end{array}$ & & \\
\hline
\end{tabular}

Fuente: (Toomey, 2009).

El desempeño efectivo de los papeles del acompañamiento a la apropiación comunitaria implica la conjugación de 7 elementos fundamentales. Primero, un mandato institucional (visión, misión, objetivos estratégicos, enfoques y estrategias) acordes, autoridades con visión y comprometidas a la horizontalidad, flexibilidad, perspectiva de largo plazo del acompañamiento y ceder el protagonismo y poder de decisión 
a los actores comunitarios. Esto potencia el establecimiento de intereses y ethos compatibles, acciones emprendidas orientadas por compromisos y objetivos compartidos, un proceso de toma de decisión conjunto y canales de comunicación claramente definidos, espacios de aprendizaje mutuo donde otros actores pueden involucrarse y nuevos temas de colaboración incorporarse, transparencia en la inversión de recursos y disfrute de los beneficios. Este involucramiento de largo plazo, está basado en el respeto, la confianza y el valor que cada uno aporta a la relación.

Segundo, la disposición de un personal de campo para la vinculación comunitaria con preparación, experiencia y una mística adecuada es indispensable. Según plantea Chambers (1983), en las comunidades, el error más generalizado es la práctica de comportamientos de dominio, superioridad, arrogancia y discriminación, producto de prejuicios o estereotipos dados por nuestra procedencia urbana, de clase, étnica o bien profesional (Chambers, 1983; 1997). Por el contrario, la práctica del desarrollo comunitario para que sea efectiva requiere de un relacionamiento con los otros en maneras que sean fraternas, empáticas, recíprocas y con propósito compartido, en un marco permanente de diálogo (Westoby \& Owen, 2010). Además, el personal de campo de vinculación comunitaria requiere una preparación para poder actuar como agente de cambio en contextos locales de conflicto o bien de asimetrías de poder e inequidad (género, generacional, étnica o económica). Pero más aún, este personal debe basar su quehacer conforme un proceso dirigido de fortalecimiento de capacidades, en el que la centralidad debe ser el aprendizaje mutuo (Kirk, 2004). Estos juegan un papel muy importante en la operacionalización del mandato institucional y en el cultivo de confianza dentro de la comunidad.

El tercer elemento es el arraigo local, comunitario del acompañamiento. En este arraigo endógeno, el actor tiene una mayor afinidad y orientación a los intereses locales (Botes \& van-Rensburg, 200o; Mintzberg \& Srinivas, 2010). Contrariamente, el arraigo exógeno está determinado por dogmas e intereses globales, acciones inducidas y gestionadas desde fuera, conforme procesos de toma de decisión de arriba hacia abajo, dominio del conocimiento experto, orientación a productos en vez de procesos y horizontes cortoplacista en la consecución de resultados. El arraigo externo es característico de organizaciones e instituciones internacionales y nacionales, tanto de gobierno, sociedad civil y cooperación al desarrollo. El arraigo endógeno es particular de organizaciones e instituciones de la misma región y localidad, cuyos marcos referenciales están vinculados a estos contextos particulares y su personal procede de la misma población.

Un cuarto elemento son las "reglas del juego" de inter-relacionamiento bien definidas. Estas pueden ser formales o tácitas, derivadas del diálogo, la negociación o bien el aprendizaje mutuo basado en experiencia colaborativa acumulada. Las "reglas del juego" definen la relación entre los actores, sus responsabilidades, potestades y expectativas (incluyendo objetivos y metas). Estas son el más importante referente en la mediación y solución de conflictos y aseguramiento de transparencia entre las partes. Estas "reglas del juego" pueden constituirse en un documento escrito, en la forma de acuerdo o convenio. En el acompañamiento planteado, estos acuerdos son también instrumento de un proceso de fortalecimiento de capacidades y especialmente de apropiación comunitaria.

Quinto, el acompañamiento a la apropiación comunitaria requiere un horizonte de tiempo adecuado que posibilite la expansión de agencia colectiva que incluye la percepción colectiva de eficacia, capital social, sentido de comunidad y capacidad de participación efectiva dentro de la comunidad, y con ello, transformaciones sostenibles. Una orientación de largo plazo permite que las comunidades incorporen sus conocimientos y capacidades endógenas en acciones colectivas tendientes a lograr cambios beneficiosos en sus vidas, avancen en su manejo integral y evaluación, tanto de las acciones y proyectos como del acompañamiento externo. Este nivel de involucramiento de la comunidad en la gestión de los procesos de desarrollo comunitario es un factor decisivo en la sostenibilidad de los mismos (Mansuri \& Rao, 2004).

Sexto, la integralidad de acciones corresponde con una estrategia de acompañamiento bien estructurada y consciente. Esta estrategia concertada con el liderazgo comunitario involucra acciones desde las dimensiones de docencia (formación, capacitación) e investigación articuladas en una plataforma de extensión o acompañamiento comunitario. En el caso de la URACCAN, los procesos de formación in- 
cluyen tanto carreras como diplomados comunitarios, investigación acción participativa con perspectiva intercultural de género en variedades de temas: educación intercultural, medicina tradicional, sistemas agroforestales, agricultura orgánica, revitalización cultural, equidad de género, seguridad alimentaria, cultura de paz, democracia y participación ciudadana, derechos humanos, co-manejo de reservas naturales, manejo forestal, conservación y uso racional de recursos naturales, demarcación territorial, prevención y gestión de riesgos, prevención de enfermedades de transmisión sexual y VIH SIDA, agua y saneamiento, mediación y negociación de conflictos, entre otros.

Y el séptimo elemento, es la intensidad en el inter-relacionamiento que está particularmente relacionado al cultivo o fortalecimiento de la agencia colectiva mediante acciones constantes. Esta implica la puesta en marcha de múltiples iniciativas- sucesivas, simultáneas e interrelacionadas en el marco de una orientación programática integral en un horizonte prudencial de tiempo. A partir de una agencia comunitaria fortalecida, las comunidades pueden emprender acción colectiva auto gestionada en frecuencia e intensidad tal que les posibilite lograr y sostener procesos de buen vivir, vivir en dignidad comunitario, en las condiciones de pobreza y exclusión.

En el acompañamiento comunitario, la apropiación comunitaria descansa en cuatro principios de trabajo fundamentales: el consentimiento, libre, previo e informado, la responsabilidad compartida, la equidad y la formación para la vida. El consentimiento previo, libre e informado trata del respeto al derecho de las comunidades indígenas, afrodescendientes y mestizas a su autodeterminación en todos los asuntos que afectan directamente sus vidas. Así, el consentimiento es producto de un diálogo horizontal entre los representantes de la Universidad y el liderazgo comunitario, que en gran medida tiene una aprobación definitiva de los acuerdos en asamblea comunitaria. Lo libre denota una transparencia y buena fe en el relacionamiento universidad-comunidad, que convierte la confianza en el principal valor de intercambio entre las partes. Lo previo está determinado por un relacionamiento de vieja data, que posibilita la confianza entre las partes. Lo informado se deriva principalmente de espacios de toma de decisión compartidos, donde la corresponsabilidad es una condición de la sostenibilidad de las acciones emprendidas.

La responsabilidad compartida o corresponsabilidad es la máxima expresión de objetivos y compromisos manifiestos y comunes a las partes, comunidad- universidad. Esta responsabilidad de las partes es ante todo moral, en cuanto denota una consciencia crítica en hacer valer los derechos y obligaciones en pro de alcanzar lo que se ha definido conjuntamente, conforme las reglas del relacionamiento establecidas. Sin embargo, a como la interculturalidad, la corresponsabilidad no está libre de conflictos. Los conflictos son indispensables en la construcción de corresponsabilidad y confianza, al depender su mediación o solución en el diálogo abierto, franco y propositivo. Por su parte, la equidad es fundamental en contrarrestar las distorsiones en la vida comunitaria expresadas principalmente en fragmentación social y desigualdad severa. En la URACCAN, el cultivo de la equidad tiene varias implicaciones como el fomento de una participación consciente y beligerante de mujeres y hombres, jóvenes y adultos de en correspondencia con su empoderamiento en cuanto a su identidad, capacidad de decisión y actuación y como parte de una comunidad (URACCAN, 2010b).

A diferencia de otros actores de desarrollo, la universidad es una fuente inagotable de formación para la vida. La formación para la vida en la URACCAN tiene varias implicaciones. Primero, el proceso de enseñanza aprendizaje debe sustentarse en procesos educativos pertinentes a las necesidades y demandas particulares del florecimiento de la comunidad, su autonomía y la intersubjetividad que la sustenta entre sus miembros. Los diplomados comunitarios son un buen ejemplo de esta perspectiva de la formación para la vida. Segundo, este proceso de enseñanza- aprendizaje es armonioso entre su teoría y su práctica, impulsado especialmente por talentos universitarios de los propios pueblos a los que pertenecen las comunidades acompañadas por la Universidad.

Así mismo, la espiritualidad facilita los procesos de acompañamiento en la apropiación comunitaria. La espiritualidad se puede entender como valores éticos y creencias que le dan significado y propósito a la existencia de las personas y grupos (Chile, 2004). La espiritualidad es un sentido de vida que interconecta 
lo individual, lo colectivo, la naturaleza y el universo, proporcionando una dirección para el logro de armonía a través del balance, la colaboración y la preocupación mutua. La espiritualidad se afirma y reafirma mediante rituales de transición, sanación y celebración, que contribuyen a dar estabilidad, continuidad y un sentido de solidaridad y cohesión en la vida comunitaria (Hustedde \& King, 2002). La espiritualidad es una fuente importante de emociones como el altruismo, el amor, la compasión y la gratitud, que sostienen la vida social y moral comunitaria. Las emociones constituyen fuerzas motrices de motivaciones y acción. Sólidos nexos emotivos se relacionan con elevados niveles de solidaridad social (Hustedde \& King, 2002).

En las comunidades de Tuara, El Carao y Españolina los grupos religiosos (Iglesia Morava e Iglesia Católica) están entre las principales organizaciones de base comunitarias, considerándose más incluyentes y democráticas en sus procesos de toma de decisión y elección. Al mismo tiempo, a estas se le reconocen un importante papel en la gestión del bienestar dentro de la comunidad. Su consideración en los procesos de acompañamiento comunitario es indispensable, especialmente porque minimizan la influencia política partidaria y promueven mayor participación de la mujer.

En la concatenación de los aspectos antes mencionados, el proceso que conlleva a la apropiación comunitaria esta mediado por factores de conversión. En las comunidades estudio de caso, existen tres factores de conversión determinando el tránsito de agencia colectiva a los niveles de participación y capital social: la influencia política partidaria, la transparencia y honestidad de los líderes y lideresas comunitarias, y el machismo institucionalizado en las estructuras comunitarias. Estos factores tienen una particular incidencia en las variables de confianza, liderazgo, acción colectiva y tipo de participación. La transparencia y honestidad de los líderes, lideresas y autoridades territoriales tienen un gran peso en estas variables, al determinar la calidad de los procesos de toma de decisión y elección en colectivo, confianza y capacidad de movilización comunitaria. La influencia política partidaria a su vez incluye en la calidad del liderazgo comunitaria, especialmente en términos de su legitimidad, sensibilidad y unidad en la comunidad. El machismo institucionalizado afecta especialmente los niveles de confianza y acción colectiva de las mujeres, teniendo un efecto a nivel general de la comunidad. 


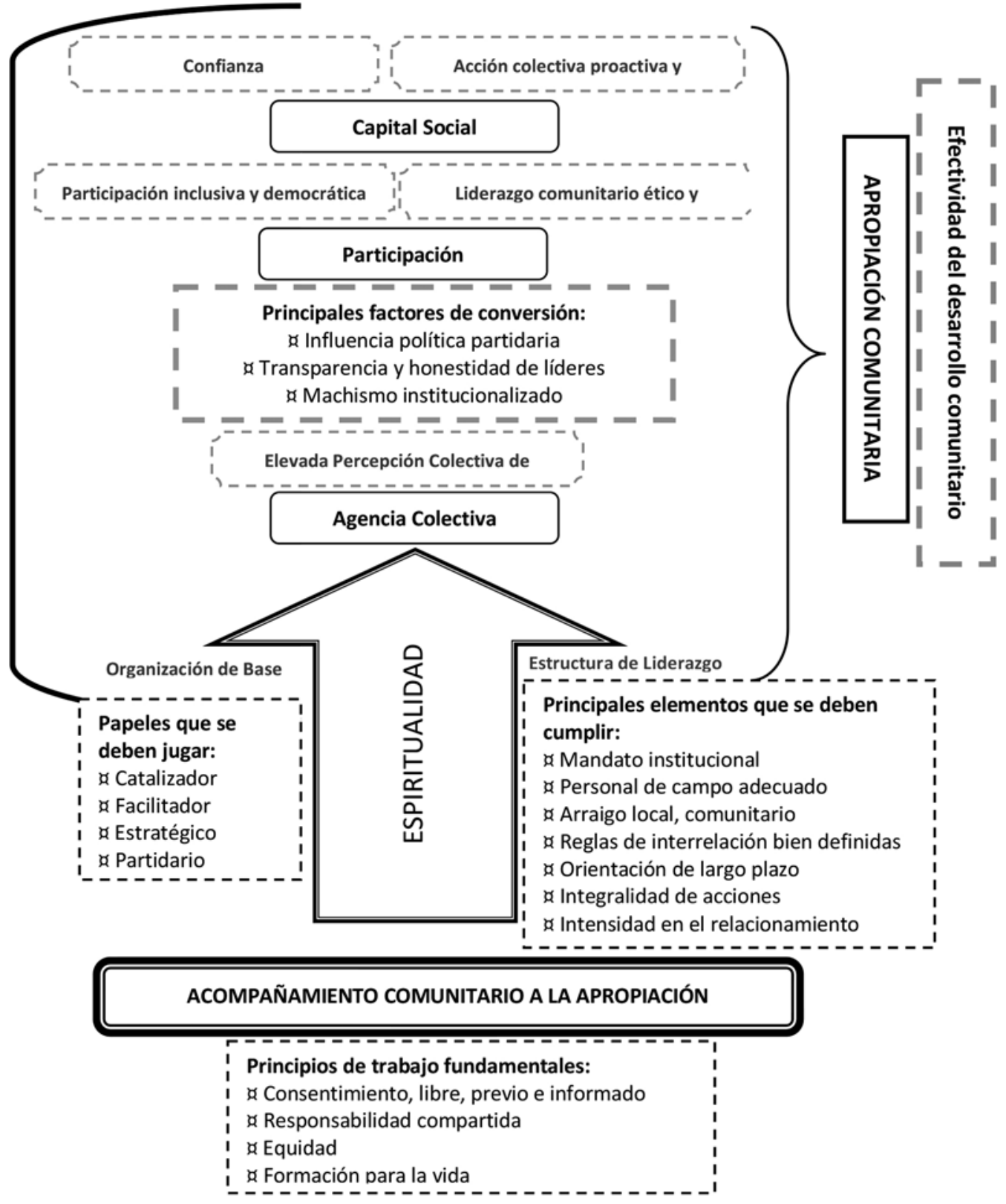

Situándolo a las Regiones Autónomas de Nicaragua, el desarrollo comunitario se puede definir como un proceso dialéctico de acompañamiento dirigido al fortalecimiento de la comunidad desde el cultivo de la agencia colectiva, intersubjetividad y participación (de empoderamiento). En este contexto, se considera que las personas tienen una predisposición epistemológica (hermenéutica) y ontológica (estructuralista) a la cultura determinar el entendimiento compartido en colectividad y las estructuras sociales a definir los alcances de su agencia, capital social y participación (Dixon et al., 2005). Los individuos son productos de sus comunidades y sus subjetividades son socialmente establecidas en su colectivo cultural, que les provee de un patrón de interpretación organizado lingüísticamente. El fortalecimiento o revitalización de la comunidad parte entonces de lo endógeno (incluyendo la identidad, valores ético-morales, conocimientos, saberes y sabidurías). Así, la efectividad del desarrollo comunitario presupone una capacidad de la comunidad de ejercer control de sus vidas y de su futuro. 
Historia y actualidad de URACCAN

\section{Conclusiones}

El marco teórico y los resultados de los estudios de caso confirman la existencia de una cadena directa de interrelación entre el acompañamiento a la apropiación comunitaria y la efectividad del desarrollo comunitario. El acompañamiento a la apropiación comunitaria es directamente proporcional a un incremento de agencia colectiva, capital social y participación, conforme sus variables de percepción colectiva de eficacia, confianza, acción colectiva, tipo de participación y nivel de liderazgo comunitario. Los niveles de apropiación colectiva comunitaria son directamente proporcionales a avances en el bienestar de la colectividad comunitaria. En los tres estudios de caso existen evidencias de esto.

La linealidad de este proceso depende en gran medida de la calidad del acompañamiento, especialmente en cuanto a su intensidad, integralidad y duración. En las comunidades estudio de caso, intervienen factores de conversión que afectan la correspondencia entre agencia colectiva, capital social y participación comunitaria, incluyendo la equidad entre mujeres y hombres. Las mujeres tienen en relación a los hombres mayor apropiación comunitaria, incluyendo sus dimensiones de agencia colectiva, participación y capital social con la excepción de Españolina, donde los hombres tienen un nivel mayor. De igual manera, las mujeres tienen mayores niveles en las variables de percepción colectiva de eficacia, nivel de liderazgo comunitario, tipo de participación con la excepción de Tuara, y Confianza en El Carao. Los hombres tienen mejores percepciones sobre nivel de acción colectiva y confianza en Tuara y Españolina.

El acompañamiento a la apropiación comunitaria es inherente al modelo de universidad comunitaria intercultural de la URACCAN, que surge como un proyecto institucional de fortalecimiento de la autonomía y del desarrollo con identidad de los pueblos y comunidades indígenas, afrodescendientes y mestizos de las Regiones Autónomas de Nicaragua. En este sentido, el mandato institucional (filosofía institucional, el marco normativo y acciones), enfoque de acompañamiento comunitario, liderazgo institucional, estructura organizacional, talentos humanos y capacidad de gestión de la Universidad son coherentes con la promoción de la apropiación comunitaria. Se debe mencionar que la subvención estatal (6\%) hace que las acciones de acompañamiento comunitario sean más costo efectivas, que las acciones de otros actores regionales especialmente de la sociedad civil.

En la medida de la calidad invertida en intencionalidad, diversidad de acciones, y duración del acompañamiento, como consecuencia de la puesta en práctica de estas ventajas y capacidades institucionales de la URACCAN, la contribución a la apropiación comunitaria arroja distintos resultados. De los tres estudios de caso, El Carao es la comunidad que ha contado con un acompañamiento de mayor intencionalidad, diversidad de acciones y durabilidad en el tiempo de parte de la Universidad. Esta calidad de acompañamiento, ha sido menor en los casos de las comunidades de Tuara y Españolina, respectivamente.

Según resultados, los participantes de las distintas actividades de acompañamiento de la URACCAN vis a vis con los restantes miembros de la comunidad encuestados presentan mayores índices de apropiación comunitaria, incluyendo índices de agencia colectiva, capital social y participación comunitaria. En relación a las variables, esta tendencia se mantiene en los índices de percepción colectiva de eficacia, confianza, tipo de participación y nivel de liderazgo comunitario. La excepción es el índice de nivel de acción colectiva. Esta tendencia se mantiene en la comunidad Españolina, con la excepción de los índices de percepción colectiva de eficacia, confianza y nivel de liderazgo comunitario. En la comunidad de Tuara, sucede lo contrario. Los comunitarios y las comunitarias que han participado en actividades de acompañamiento de la Universidad reflejan menores percepciones de apropiación comunitaria y de sus variables con la excepción de agencia colectiva. Esta diferencia tiene su explicación en la pérdida de integralidad del acompañamiento comunitario.

Desde el 2006, el acompañamiento se ha centrado en pocos temas como demarcación y titulación de la propiedad comunal, ordenamiento forestal y forestería comunitaria. Estos temas tienden a una menor movilización de la participación y acción colectiva y un mayor efecto en la agencia colectiva al estar centrado en cuestiones técnico legales, la estructura de liderazgo de la comunidad y pocas familias. Un segundo aspecto a señalar es el tránsito hacia un acompañamiento multi-comunal y multi-territorial de 
menor alcance. De esta manera, el acompañamiento a la comunidad de Tuara ha dejado de ser exclusivo, diluyéndose en su intensidad y duración. En este mismo sentido, el acompañamiento de la Universidad lo realiza principalmente una sola instancia del Recinto Bilwi: el Instituto de Recursos Naturales, Medio Ambiente y Desarrollo Sostenible (IREMADES).

Por otra parte, en las comunidades indígenas y mestizas, la etnicidad pudiera explicar, más que la duración del acompañamiento una mayor contribución del acompañamiento de la Universidad al capital social y la participación dado los temas abordados que están conforme los antecedentes de vida en colectividad de las comunidades indígenas y sus demandas históricas de reivindicación de derechos colectivos. La intensidad de acciones y duración podrían explicar una mayor agencia colectiva en la comunidad de El Carao.

En las tres comunidades de estudio de caso, se identifican la influencia política partidaria en las estructuras de liderazgo, el abuso del alcohol, las drogas y el robo. Las comunidades indígenas tienen también una tensión con la invasión de sus territorios comunales por colonos o invasores mestizos y la pérdida de valores y prácticas culturales de las nuevas generaciones. En la comunidad mestiza de El Carao el cambio climático a consecuencia del acelerado despale de los bosques y la violencia familiar y social, que es una herencia de la guerra de los 8o's, son factores fundamentales.

En las comunidades estudio de caso se observan tres etapas diferentes en el acompañamiento de la apropiación comunitaria: una etapa inicial, una etapa intermedia y una etapa alta de apropiación comunitaria. En la etapa inicial, el índice de apropiación está a un nivel menor al 50\%. Aunque exista una estrategia de acompañamiento bien estructurada y consciente permitiendo elevar la agencia colectiva, el capital social y la participación presentan niveles importantes de rezago para su potenciación en el desarrollo comunitario. Los bajos niveles de confianza, la acción colectiva y el nivel de liderazgo comunitario son aspectos claves en este rezago. En la etapa intermedia la apropiación alcanza niveles que van de un $51 \%$ a $79 \%$. En esta etapa existe un nivel superior de agencia colectiva, pero aun no corresponden con niveles elevados de capital social y participación. La confianza, acción colectiva y el liderazgo comunitario son variables claves en esto. En esta etapa, la estrategia de acompañamiento puede ser estructurada y consciente, además de diversa e intensa en sus acciones y de duración prolongada. La diferencia entre estas dos etapas está en las bases de solidaridad o valores de colectividad histórica o culturalmente presentes o predispuestos en la comunidad.

La etapa alta de apropiación comunitaria, que no ha coincidido con las tres comunidades estudio de caso, representa el mayor nivel que se puede alcanzar en las dimensiones y variables de la apropiación comunitaria. El índice de apropiación comunitaria corresponde con valores oscilando entre $80 \%$ a $100 \%$. En esta etapa, se tiene unas estrategias de acompañamiento estructurada, consciente, diversas, intensas y de prolongada duración tanto dirigidas a la agencia colectiva, pero también a fortalecer el capital social y la participación. Desde luego, también significa una atención consciente a los factores de conversión.

Finalmente, este proceso investigativo aspiró a identificar indicadores de impactos para la medición de la apropiación comunitaria en el contexto multicultural de las Regiones Autónomas de Nicaragua. Al respecto, se han identificado los siguientes indicadores:

Cuadro 3: Indicadores utilizados en la estimación de la apropiación comunitaria

\begin{tabular}{|l|l|l|l|l|}
\hline \multicolumn{1}{|c|}{$\begin{array}{c}\text { Percepción Colectiva de } \\
\text { Eficacia }\end{array}$} & \multicolumn{1}{|c|}{ Confianza } & $\begin{array}{c}\text { Nivel de Acción } \\
\text { Colectiva }\end{array}$ & $\begin{array}{c}\text { Tipo de Participación } \\
\text { Comunitaria }\end{array}$ & $\begin{array}{c}\text { Nivel de Liderazgo Comu- } \\
\text { nitario }\end{array}$ \\
\hline $\begin{array}{l}\text { Sentido de derechos como poder den- } \\
\text { tro de la comunidad. }\end{array}$ & $\begin{array}{l}\text { Confianza en la mayoría de las } \\
\text { personas que viven en la comu- } \\
\text { nidad. }\end{array}$ & $\begin{array}{l}\text { Trabajo en beneficio de la } \\
\text { comunidad. }\end{array}$ & $\begin{array}{l}\text { Como se toman las decisiones en } \\
\text { las 2 principales organizaciones de } \\
\text { base comunitarias. }\end{array}$ & $\begin{array}{l}\text { Consideración de los líderes comu- } \\
\text { nitarios de los problemas de los } \\
\text { miembros de la comunidad al tomar } \\
\text { decisiones. }\end{array}$ \\
\hline
\end{tabular}




\begin{tabular}{|l|l|l|l|l|}
\hline \multicolumn{1}{|c|}{$\begin{array}{c}\text { Percepción Colectiva de } \\
\text { Eficacia }\end{array}$} & \multicolumn{1}{|c|}{ Confianza } & \multicolumn{1}{c|}{$\begin{array}{c}\text { Nivel de Acción } \\
\text { Colectiva }\end{array}$} & \multicolumn{1}{c|}{$\begin{array}{c}\text { Tipo de Participación } \\
\text { Comunitaria }\end{array}$} & $\begin{array}{c}\text { Nivel de Liderazgo Comu- } \\
\text { nitario }\end{array}$ \\
\hline Unidad en comunidad. & $\begin{array}{l}\text { Confianza en los líderes y } \\
\text { lideresas de la comunidad. }\end{array}$ & $\begin{array}{l}\text { Cantidad de participación en } \\
\text { actividades de la comunidad. }\end{array}$ & $\begin{array}{l}\text { Como se seleccionan los líderes en } \\
\text { las } 2 \text { principales organizaciones de } \\
\text { base comunitarias. }\end{array}$ & $\begin{array}{l}\text { Honestidad de los líderes comuni- } \\
\text { tarios. }\end{array}$ \\
\hline $\begin{array}{l}\text { Unidad de comunidad en presentar } \\
\text { visión y prioridades ante actores ex- } \\
\text { ternos. }\end{array}$ & $\begin{array}{l}\text { Confianza en las autori- } \\
\text { dades del gobierno terri- } \\
\text { torial. }\end{array}$ & $\begin{array}{l}\text { Crítica o sanción por la no } \\
\text { participación en actividades } \\
\text { de la comunidad. }\end{array}$ & $\begin{array}{l}\text { Como es el liderazgo en las 2 prin- } \\
\text { cipales organizaciones de base co- } \\
\text { munitarias. }\end{array}$ & $\begin{array}{l}\text { Honestidad de las autoridades terri- } \\
\text { toriales. }\end{array}$ \\
\hline $\begin{array}{l}\text { Control de la comunidad ante actores } \\
\text { e instituciones externas sobre decisio- } \\
\text { nes de asuntos que afectan sus vidas. }\end{array}$ & $\begin{array}{l}\text { Confianza en los técnicosy } \\
\text { funcionarios de URACCAN. }\end{array}$ & $\begin{array}{l}\text { Cantidad de personas que } \\
\text { participan en actividades } \\
\text { organizadas por los líderes } \\
\text { comunitarios. }\end{array}$ & $\begin{array}{l}\text { Honestidad del personal de URAC- } \\
\text { CAN. }\end{array}$ & $\begin{array}{l}\text { Mejora, mantenimiento o deterioro } \\
\text { de la honestidad de los líderes co- } \\
\text { munitarios. }\end{array}$ \\
\hline $\begin{array}{l}\text { Compromiso de miembros de la comu- } \\
\text { nidad con objetivos comunes a pesar } \\
\text { de diferencias existentes. }\end{array}$ & $\begin{array}{l}\text { Como se llevan las perso- } \\
\text { nas en la comunidad. }\end{array}$ & $\begin{array}{l}\text { Movilización comunitaria } \\
\text { para ayuda en casos de enfer- } \\
\text { medad o muerte. }\end{array}$ & \\
\hline
\end{tabular}

\section{Lista de referencias}

Alkire, S. (2008). Concepts and measures of agency. OPHI Working Paper (9), 1- 23.

Bandura, A. (1988). Organisational applications of social cognitive theory. Australian Journal of Management, 13(2), 275-302.

Bell, J. (2002). Cómo hacer tu primer trabajo de investigación. Guía para investigadores en educación y ciencias sociales. Barcelona, España: Gedisa Editorial.

Botes, L., \& van-Rensburg, D. (2000). Community participation in development: Nine plagues and twelve commandments. Community Development Journal, 35(1), 41-58.

Bowen, G. A. (2008). An analysis of citizen participation in anti- poverty programmes. Community Development Journal, 43(1), 65-78.

Butterfoss. (2006). Process evaluation for community participation. Annual Reviews in Public Health(27), 323-340.

Cohen, A. P. (1985). The symbolic construction of community. In P. Hamilton (Series Ed.) Key ideas, (pp. 2206).

Cronick, K. (2002). Community, Subjectivity, and Intersubjectivity. American Journal of Community Psychology, 30(4), 529-546. doi: 10.1023/a:1015860002096

Chambers, R. (1983). Rural development. Putting the last first. London: Pearson Education Longman Limited.

Chambers, R. (1997). Whose reality counts? Putting the first last. London: Intermediate Technology Publications.

Chile, L. M. (2004). Spirituality and community development: Exploring the link between the individual and the collective. Community Development Journal, 39(4), 318-331. doi: 10.1093/cdj/bsho29 
Dahl- Ostergaard, T., Moore, D., Ramirez, V., Wenner, M., \& Bonde, A. (2003). Desarrollo rural de autogestión comunitaria ¿Qué hemos aprendido? (D. D. Sostenible, Trans.) Informes Técnicos (pp. 75). Washington, D.C.: Banco Interamericano de Desarrollo (BID).

De Silva, M. J., Harpham, T., Huttly, S. R., Bartolini, R., \& Penny, M. E. (2007). Understanding sources and types of social capital in Peru. Community Development Journal, 42(1), 19- 32.

Dixon, J., Dogan, R., \& Sanderson, A. (2005). Community and communitarianism: A philosophical investigation. Community Development Journal, 40(1), 4-16. doi: 10.1093/cdj/bsioo2

Flyvbjerg, B. (2007). CInco malentendidos acerca de la investigación mediante los estudios de caso. Revista Española de Investigaciones Sociológicas(106), 33-62.

Geoghegan, M., \& Powell, F. (2009). Community development and the contested politics of the late modern agora: of, alongside or against neoliberalism?. Community Development Journal, 44(4), 430- 447.

Hustedde, R., \& King, B. (2002). Rituals: emotions, community faith in soul and the messiness of life. Community Development Journal, 37(4), 338-348.

Kirk, P. (2004). Community leadership development. Community Development Journal, 39(3), 234-251. doi: $10.1093 / \mathrm{cdj} / \mathrm{bsho19}$

Lev-Wiesel, R. (2003). Indicators constituting the construct of 'perceived community cohesion'. Community Development Journal, 38(4), 332-343.

Mansuri, G., \& Rao, V. (2004). Community- based and- driven development: A critical review. Policy Research Working Paper (pp. 77). Washington, D.C.: World Bank.

Mintzberg, H., \& Srinivas, N. (2010). Juxtaposing doers and helpers in development. Community Development Journal, 45(1), 39-57.

Miranda, F., \& Monzo, E. (2003). Capital social, estrategias individuales y colectivas: el impacto de programas públicos en tres comunidades campesinas de Chile. Santiago de Chile: CEPAL.

Nkansa, G. A., \& Chapman, D. W. (2006). Sustaining Community Participation: What Remains After the Money Ends? International Review of Education, 52(6), 509-532. doi: 10.1007/s11159-006-9009-9

O'Donnell, C. R. (2006). Beyond Diversity: Toward a Cultural Community Psychology. American Journal of Community Psychology, 37(1-2), 1-7. doi: 10.1007/s10464-005-9010-7

Sampieri, R. H., Fernández-Collado, C., \& Lucio, P. B. (2006). Metodología de la investigación (Cuarta edición ed.). México, D.F., México: McGraw Hill.

Sen, A. (1999). Development as freedom. Oxford: Oxford University Press.

Toomey, A. H. (2009). Empowerment and disempowerment in community development practice: eight roles practitioners play. Community Development Journal, 46(2), 181-195. doi: 10.1093/cdj/bspo6o

URACCAN. (2010). Política intercultural de género de URACCAN (pp. 29). Bluefields, RAAS, Nicaragua: Centro de Estudios e Información de la Mujer Multiétnica (CEIMM), Universidad de las Regiones Autónomas de la Costa Caribe Nicaragüense (URACCAN). 
Urquijo Angarita, M. J. (2007). El enfoque de las capacidades de Amartya Sen: alcance y límites. (Doctorado en Ética y Democracia Tésis Doctoral), Universidad de Valencia, Valencia.

Unceta Satrústegui, K. (2007). La cooperación al desarrollo en las universidades españolas. Madrid, España: Agencia Española de Cooperación Internacional (AECID), Ministerio de Asuntos Exteriores y de Cooperación.

Westoby, P., \& Owen, J. (2010). The sociality and geometry of community development practice. Community Development Journal, 45(1), 58-74.

Yacuzzi, E. (2005). El estudio de caso como metodología de investigación: Teoría, mecanismos causales, validación, from http://www.automark.com.mx/MYRNA_estudiosdecaso.pdf

Yin, R. K. (2003) Application of case study research. Applied social research methods series (Second edition ed., pp. 1-153). Thousand Oaks, California: SAGE Publications. 